\title{
IRIS Recognition: Review and Open Issues
}

\author{
Rajesh R. Karhe \\ Associate Professor, \\ Depart of Electronics and Commmunication, \\ Shri Gulabrao Deokar College of Engineering, Jalgaon. \\ Madhuri Waman Patil \\ Depart of Electronics and Commmunication, \\ Shri Gulabrao Deokar College of Engineering, Jalgaon.
}

\begin{abstract}
Among the various biometrics technologies, iris recognition is the most reliable and accurate biometric identification system available. Iris Recognition using the features of retinal microvascular network is the new area of research. The iris recognition systems have made tremendous progress over the past decade, but further work remains to improve their accuracy in environments characterized by unfavorable lighting, large stand-off distances, and moving subjects. For the purpose of research and development of Iris recognition technology there are few public and freely available databases to have sample images. These iris databases contributes rich amount of iris images which were taken in different environments. In this paper we will discuss about the iris anatomy, history, general process, various implemented locations of system at international level, international competitions and publicly available iris image datasets.
\end{abstract}

Index Terms—biometric system, Iris recognition, segmentation, feature extraction

\section{INTRODUCTION}

The need for infallible security systems has become a vital aspect in public security. Iris recognition is one of the important techniques and compared with other biometric features (such as face, voice, etc.), the iris is more stable and reliable for identification [1]. Iris recognition is the analysis of the coloured ring that surrounds the pupil [1]. The iris has unique structure and these patterns are randomly distributed, which can be used for identification of human being.

Retina: In the center of the retina is the optic nerve, a circular to oval white area measuring about $2 \times 1.5 \mathrm{~mm}$ across. From the center of the optic nerve radiates the major blood vessels of the retina.

Approximately 17 degrees $(4.5-5 \mathrm{~mm})$, or two and half disc diameters to the left of the disc, can be seen the slightly oval-shaped, blood vessel-free reddish spot, the fovea, which is at the center of the area known as the macula by ophthalmologists

Unlike traditionally used biometric systems, vascular pattern of human retina is the most reliable and stable source for biometrics. It is not easy to forge it as it lies at back end of human eye and is not directly accessible [2]. There is always a confusion regarding iris and retina as they both belong to human eye but their functions and patterns are completely different. Iris is the colored region between the pupil and sclera whereas retina is located at back region of eye. The foundation of retinal recognition is the pattern of blood vessels present in human retina [2]. Figure 1 shows the human retinal and vascular pattern extracted from digital retinal image.

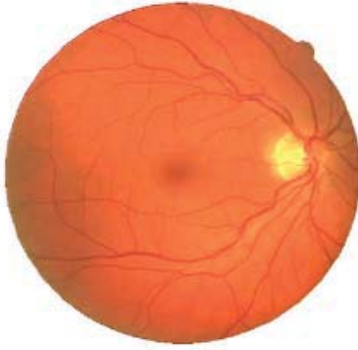

(a)

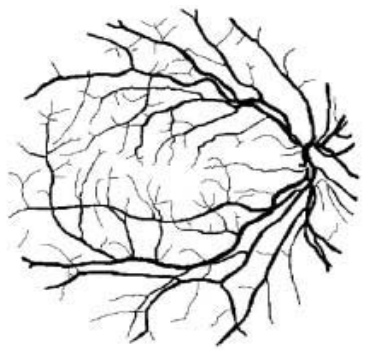

(b)

Figure 1 a. Digital Image of Human Retina b. Vascular Pattern 
In today's networked world, the need to maintain the security of information or physical property is becoming both increasingly important and difficult. Most of the times, criminals have been taking advantage of a fundamental flaw in the conventional access control systems. The access control systems based on biometrics have a potential to overcome most of the deficiencies of current security systems and have been gaining importance in recent years.

\section{RELATED WORK FOR NOISE REMOVAL}

Image noise is the random variation of brightness or color information in images produced by the sensor and circuitry of a scanner or digital camera. Image noise can also originate in film grain and in the unavoidable shot noise of an ideal photon detector [3].Image noise is generally regarded as an undesirable by-product of image capture. Although these unwanted fluctuations became known as "noise" by analogy with unwanted sound they are inaudible and such as dithering. The types of Noise are Amplifier noise (Gaussian noise), Saltand-pepper noise, Shot noise (Poisson noise), Speckle noise.

Image de-noising is very important task in image processing for the analysis of images. Ample image denoising algorithms are available, but the best one should remove the noise completely from the image, while preserving the details. Broadly speaking, De-noising filters can be classified in the following categories:

\section{A. Mean Filter}

Mean filter is an averaging linear filter. Here the filter computes the average value of the corrupted image in a predefined area. Then the center pixel intensity value is replaced by that average value. This process is repeated for all pixel values in the image.

The algorithm proposed in [4] considers first order neighborhood pixels for detecting the noisy pixel and mean filter is considered. Color images are also de-noised by extracting the R, G and B pixels from noisy image and then they are de-noised separately and then merged together to again form the color image. The algorithm is compared with all other standard and well known algorithms and found to have good noise removal capabilities at high densities.

In order to improve the speed of mean filter algorithm and to reduce the redundancy operation, an efficient algorithm is presented in [5]. At first, an assistant array is built up to store the pixels' value summation of each column. Then, according to the relationship between adjacent windows when filter window is siding along the rows, a recursive formula for calculating the midpoint mean of a new window is schemed out. Meanwhile, the recursive formula for updating assistant array items is obtained based on the relationship between adjacent rows' pixels in the changing row process of midpoint of window. On the basis of these two recursion formulas, a high efficient mean filter algorithm is achieved.

\section{B. Median Filter}

The Median filter is a nonlinear digital filtering technique, often used to remove noise. Such noise reduction is a typical preprocessing step to improve the results of later processing (for example, edge detection on an image). Median filtering is very widely used in digital image processing because under certain conditions, it preserves edges whilst removing noise. The main idea of the median filter is to run through the signal entry by entry, replacing each entry with the median of neighboring entries. Note that if the window has an odd number of entries, then the median is simple to define: it is just the middle value after all the entries in the window are sorted numerically. For an even number of entries, there is more than one possible median. The median filter is a robust filter. Median filters are widely used as smoothers for image processing, as well as in signal processing and time series processing. A major advantage of the median filter over linear filters is that the median filter can eliminate the effect of input noise values with extremely large magnitudes.

Authors in [6] proposes a novel decision-based median filter that replaces each corrupted pixel with the median value of their $k$-nearest noise-free pixels. Advantages of the median filter using $k$-nearest noise-free pixels instead of $k$-nearest pixels are two facets: first, it guarantees that pixels after being restored must be noise-free, because the median filter operator is executed on noise-free pixels; second, the median filter using $k$-nearest noise-free pixels adaptively adjusts its window size for each pixel such that the number of noise-free pixels locating in the window increases up to $k$. To realize it, the median filter using $k$-nearest noise-free pixels firstly 
detects noise-free pixels in an image, then replaces each corrupted pixel with the median value of their knearest noise-free pixels.

[7] Presents the removal of high density salt and pepper noise in gray scale Images using fuzzy based median filter (FBMF) algorithm. FBMF replaces the noisy pixel by median value when O's, 255's and other pixel values are present in the chosen window and when all pixel values are either O's or 255's, or combination of these, then the noise pixel is replaced by fuzzy membership value of a selected window.

\section{Wiener Filter}

The goal of the Wiener filter is to filter out noise that has corrupted a signal. It is based on a statistical approach. Typical filters are designed for a desired frequency response. The Wiener filter approaches filtering from a different angle. One is assumed to have knowledge of the spectral properties of the original signal and the noise, and one seeks the LTI filter whose output would come as close to the original signal as possible.

A new widely linear noise-reduction Wiener filter based on the variance and pseudo-variance of the short-time Fourier transform coefficients of speech signals is proposed in [8]. Authors show that this

new noise-reduction filter has many interesting properties, including but not limited to: 1) it causes less speech distortion as compared to the classical noise-reduction Wiener filter; 2) its minimum mean squared error (MSE) is smaller than that of the classical Wiener filter; 3) it can increase the subband signal-to-noise ratio (SNR), while the classical Wiener filter has no effect on the subband SNR for any given signal frame and subband.

An adaptive wiener filter design method is presented in [9] using quality based hybrid algorithms. The proposed method restore degraded image using Wiener filter with Gradient based smoothing and Median filter with an adaption of Bilateral filter for edge preserving and Guided filter for reducing staircase effect and gradient reversal. Using this method, clear images are obtained from degraded images without increasing noise, ringing artifacts and halo effect.

\section{Wavelet Transform}

In several applications, it might be essential to analyze a given signal. The structure and features of the given signal may be better understood by transforming the data into another domain. There are several transforms available like the Fourier transform, Hilbert transform, wavelet transform, etc. The Fourier transform is probably the most popular transform. However the Fourier transform gives only the frequency-amplitude representation of the raw signal. The time information is lost. So we cannot use the Fourier transform in applications which require both time as well as frequency information at the same time. The Short Time Fourier Transform (STFT) was developed to overcome this drawback.

According to the characteristic of river sediment image, the two-dimensional image data is transformed into one-dimensional array data from horizontal, vertical, + diagonal line, - the diagonal line four directions, by using different threshold value on the different wavelet decomposition high frequency coefficient and enhancing the processed coefficient, de-noise image is obtained after inversing wavelet transform and calculating on four two-dimensional pictures by unequal coefficient. The results indicate the proposed algorithm in [10] is effective both in reserving the edge and in removing noise.

Authors in [11] have proposed an improved wavelets shrinkage

method that uses different parameters across different wavelet scales to denoise the Besov images and it outperforms the existing á fixed algorithms. It is obvious that different parts of an image or different images have different structures and properties, so we assume that these differences could be described by means of their distinct smoothness properties. In experiments, authors used three simple mathematic functions to denote the relationship between the smoothness index and wavelet scale and found that the linear function achieved a better denoising effect.

\section{E. Order Statistics Filter}

Order-Statistics filters are non-linear filters whose response depends on the ordering of pixels encompassed by the filter area. When the center value of the pixel in the image area is replaced by 100th percentile, the filter is 
called max-filter. On the other hand, if the same pixel value is replaced by 0th percentile, the filter is termed as minimum filter.

Directional order statistics filtering approaches are performance limited owing to their implementation with larger window sizes (to a maximum size of 9x9) and with the need of iterative operation to filter the residual noise for highly corrupted images. Authors in [12] presents a methodology to improve the performance of directional order statistics filters for suppression of salt-and-pepper noise. The proposed filtering approach involves eight directions to identify boundary and no boundary pixels. If the non-boundary pixels are noise free, they are left unprocessed otherwise; updated by order statistics parameters of the local window.

A theoretical study and experimental validation on a binaural hearing aid setup of this standard SDW-MWF implementation is presented in [13], where the effect of estimation errors in the second-order statistics is analyzed. In this case, and for a single target speech source, the standard SDW-MWF implementation is found not to behave as predicted theoretically. Second, two recently introduced alternative filters, namely the rank-one SDW-MWF and the spatial prediction SDW-MWF, are also studied in the presence of estimation errors in the second-order statistics. These filters implicitly assume a single target speech source, but still only rely on the speech and noise correlation matrices. It is proven theoretically and illustrated through experiments that these alternative SDW-MWF implementations behave close to the theoretical optimum, and hence outperform the standard SDW-MWF implementation.

\section{FEATURE EXTRACTION RELATED WORK}

Li et. al., introduced a new method to detect vascular bifurcations and crossovers in fundus images. The Gaussian filter is applied to the blue channel of the original color retinal images to suppress the central reflex and reduce the candidate points. The eigenvalues and eigenvectors of Hessian matrix are then obtained in multiple scales to provide the structural and directional information. By computing the anisotropy and isotropy of neighboring image segments for each pixel in a retinal image, a multi-scale vessel filter is defined which combines the responses of tubular structures and the responses of bifurcations and crossovers [32].

Azzopardi and Petco, proposed a method for automatic detection of vascular bifurcations in segmented retinal images using trainable COSFIRE filters. The vascular tree observed in a retinal fundus image can provide clues for cardiovascular diseases. Its analysis requires the identification of vessel bifurcations and crossovers. Authors use a set of trainable key point detectors that are called as Combination of Shifted Filter Responses or COSFIRE filters to automatically detect vascular bifurcations in segmented retinal images [33].

Bhuiyan et. al., introduced a method to detect and classify the vascular bifurcation, branch and crossover points (landmarks) based on the vessel geometrical features. They utilize the vessel's centerline and width information to detect and classify these landmarks, which can be used for image matching in medical diagnosis and biometric security applications. The geometrical properties of the blood vessels passing through the potential landmarks are obtained. Perceptual grouping and Support Vector Machine (SVM) are used to classify the landmarks into the vascular bifurcations, branches and crossovers [34].

Ardizzone et. al., presents an effective algorithm for automated extraction of the vascular tree in retinal images, including bifurcations, crossovers and endpoints detection. Correct identification of these features in the ocular fundus helps the diagnosis of important systematic diseases, such as diabetes and hypertension. The preprocessing consists in artifacts removal based on anisotropic diffusion filter. Then a matched filter is applied to enhance blood vessels. The filter uses a full adaptive kernel because each vessel has a proper orientation and thickness. The kernel of the filter needs to be rotated for all possible directions. As a consequence, a suitable kernel has been designed to match this requirement. The maximum filter response is retained for each pixel and the contrast is increased again to make easier the next step. A threshold operator is applied to obtain a binary image of the vascular tree [35].

\section{PUBLICLY AVAILABLE IRIS DATABASES}

\section{A. Bath Database [36]}

The University of Bath (BATH) iris image database is constantly growing and at present contains over 16000 iris images taken from 800 eyes of 400 subjects. It results of a project which aims to build a "high quality iris image resource". The majority of the database comprises images taken from students and staff of the University of Bath the images are of very high quality, taken with a professional machine vision camera, 
mounted on a height-adjustable camera-stand. The illumination was provided through an array of infrared LEDs, positioned below the camera and set at an angle such that reflections were restricted to the pupil. Further, an infrared pass filter was used in order to cut out the daylight and other environmental light reflections on the irises region. So, this framework increases the images quality, while turned it less appropriate for the testing of iris recognition method.

\section{B. CASIA Database[37]}

Iris recognition has been an active research topic of the Institute of Automation from the Chinese Academy of Sciences. Having concluded about a lack of iris data for algorithm testing, they developed the CASIA image database. CASIA iris image database (version 1.0, the only one that we had access to) includes 756 iris images from 108 eyes, hence 108 classes. For each eye, 7 images are captured in two sessions, where three samples are collected in the first and four in the second session. Similarly to the above described database, its images were captured within a highly constrained capturing environment, which conditioned the characteristics of the resultant images. They present very close and homogeneous characteristics and their noise factors are exclusively related with iris obstructions by eyelids and eyelashes. Moreover, the post process of the images filled the pupil regions with black pixels, which some authors used to facilitate the segmentation task. So, this significantly decreased the utility of the database in the evaluation of robust iris recognition methods.

\section{ICE Database}

The Iris Challenge Evaluation (ICE) is a contest designed to measure the accuracy of the underlying technology that makes iris recognition possible. It is divided into two stages: first, it was asked to researchers and developers to participate in "iris recognition challenge problems" that might improve their recognition algorithms. Later, an opportunity to participate in a large-scale and independent evaluation will be given, through a new iris data set and a proper evaluation framework.

The ICE database is comprised of 2954 images, with a variable number of images per subject. Similarly to the remaining public iris databases, its images were captured having quality as the main concern and clearly simulate the users' cooperation in the image capturing. Therefore, the noise factors that the ICE database contains are almost exclusively related with iris obstructions and poor focused images. Interestingly, there are some images that were deliberately rotated. Also, some irises were partially captured.

\section{MMU Database[38]}

The Multimedia University has developed a small data set of 450 iris images (MMU). They was captured through one of the most common iris recognition cameras presently functioning (LG Iris Access 2200). This is a semi-automated camera that operates at the range of 7-25 cm. Further, a new data set (MMU2) comprised of 995 iris images has been released and another common iris recognition camera (Panasonic BM-ET100US Authenticam) was used. The iris images are from 100 volunteers with different ages and nationalities. They come from Asia, Middle East, Africa and Europe and each of them contributed with five iris images from each eye. Obviously, the images are highly homogeneous and their noise factors are exclusively related with small iris obstructions by eyelids and eyelashes

\section{E. UPOL Database[39]}

The UPOL iris image database was built within the University of Palack'eho and Olomouc. Its images have the singularity of being captured through an optometric framework (TOPCON TRC50IA) and, due to this, are of extremely high quality and suitable for the evaluation of iris recognition in completely noise-free environments. The database contains 384 images extracted from both eyes of 64 subjects (three images per eye). As can be seen in figure 2.5, its images have maximum homogeneity and inclusively the iris segmentation is facilitated by the dark circle that surrounds the region corresponding to the iris. Obviously, these characteristics make this database the less appropriate for the non-cooperative iris recognition research.

\section{F. WVU Database}

The West Virginia University 5 developed an iris image database (WVU) comprised of 1852 images from 380 different eyes. The number of acquisitions from each eye ranges between three and six and an OKI IrisPass-H hand-held device was used. Images of the WVU database were captured with less constraining imaging conditions and, due to this, incorporate several types of noise, such as iris obstructions, poor focused and offangle iris images. However, there are few iris images with significant regions affected by specular and lighting reflections, which we believe to be the most common type of noise resultant of natural light imaging environments 


\section{G. UBIRIS Database[40]}

After studying the above described iris databases, we concluded that none of them was suitable for the evaluation of robust iris recognition methods, those where noise identification and handling assumes higher relevance. Apart from the WVU database, that contains some noise factors but significantly lacks iris secular and lighting reflections, all the remaining databases were constructed within cooperative environments. This makes them more suitable for the preliminary evaluation of iris segmentation, feature extraction or comparison strategies, when the noise factors constitute an a priori obstacle to conclude about their merits.

Based on this, we decided to build a new public and freely available iris images database - UBIRIS - with a fundamental characteristic that distinguished it from the remaining ones: it is a "noisy iris image database" and the noise factors are not only avoided but rather induced, in order to simulate the non-cooperative image capturing. UBIRIS database is comprised of 1877 images collected from 241 subjects within the University of Beira Interior 6 in two distinct sessions and constituted, at its release date, the world's largest public and free iris database for biometric purposes.

\section{H. IIT Delhi Database[41]}

The IIT Delhi Iris Database mainly consists of the iris images collected from the students and staff at IIT Delhi, New Delhi, India. This database has been acquired using JIRIS, JPC1000 and digital CMOS camera in Biometrics Research Laboratory during Jan - July 2007 (still in progress). The image acquisition program was written to acquire and save these images in bitmap format and is also freely available on request. The currently available database consists of images taken from 224 users which are in bitmap (*.bmp) format. All the subjects in the database are in the age group 14-55 years comprising of 176 males and 48 females. The database consists of 1120 images which is organized into 224 different folders each associated with the integer identification/number. All these images were acquired in indoor environment having resolution of images 320 x 240 pixels.

\section{RELATED WORK ON IRIS RECOGNITION}

A lot of work has been done in the field of biometric iris recognition and some of the research articles discussed in this section.

Daugman [40] proposed first working methodology related to the iris biometrics. In this, Daugman makes use of an integro-differential operator for locating the circular iris and pupil region, and also the arcs of the upper and lower eyelids. The operator searches for the circular path where there is maximum change in pixel values, by varying the radius and centre $\mathrm{x}$ and $\mathrm{y}$ position of the circular contour. The operator is applied iteratively with the amount of smoothing progressively reduced in order to attain precise localization. Eyelids are localized in a parallel manner. The integro-differential can be seen as a variation of the Hough transform, since it too use of first derivatives of the image and performs a search to find geometric parameters. Since it works with raw derivative information, it does not suffer from thresholding problems of the Hough transform. However, the algorithm can fail where there is noise in the eye image, such as from reflections, since it works only on a local scale.

Daouk et al. [41] proposed iris recognition schemes which involve a fusion mechanism of both a Canny Edge Detection scheme and a Circular Hough Transform, to detect the iris's boundaries in digital image of an eye. Then Haar wavelet is used in order to pull out the deterministic patterns in a person's iris in the form of a feature vector. Wavelet tree was utilized for image coefficient's mapping where a database of 60 pictures was used and average correct recognition rate is $93 \%$. The limitation of this work is that this methodology does not perform well in the occurrence of bad lighting, occlusion by eyelids, noises or inappropriate eye positioning

Noh et al. [42] introduced a new technique of feature extraction where in this instead of using wavelet transform an adaptive method of feature extraction was introduced in which two types of Global and Local features were extracted from wavelet coefficient. Polar coordinates system was used for the mathematical modeling of the system. Global features are invariant to the eye image rotation and the imprecise iris localization. The customized geometric moment is used for representing global iris feature. Local features provide precise information regarding iris. The main reason to introduce this approach was the absence of shift-invariant property in Discrete Wavelet Transform (DWT) and the methodology which does not include shift-invariant property can't provide exact texture analysis. 
$\mathrm{Xu}$ et al. [4] proposed an improved iris recognition system which deals with the eyelids and eyelashes detection and an alternative image enhancement method. The main reason for considering eyelids and eyelashes detection is that the presence of these affects the iris image and produce noise that results in the degradation of system performance. Subblock of eyelids/eyelashes models compared for detection purpose. For enhancement of iris image subtraction of background was done. Filtering is performed by histogram equalizing and viener filtering. For eyelids/eyelashes detection summary derivate was used. The iris location finding rate is $98.42 \%$ in case of CASIA database.

Azizi et al. [43] proposed a work which deals with using features extraction and subset selection. Iris features were extracted using contourlet transform; it captures the intrinsic geometrical structures of iris image. Iris image was further decomposed into sub-blocks that contain all texture information. This technique utilizes Support Vector Machine (SVM) for matching the iris templates. Gabor filter and Haar wavelet were used in this work for coding purpose. Iris vector was created using Principal Component Analysis (PCA). The performance of the proposed system was checked against CASIA image database

Gupta and Saini [44] evaluated the existing performance of iris recognition systems by using Matlab Image Processing Toolbox. The proposed technique consisted of several basic step including image acquisition, segmentation (to detect circles of pupil and iris boundary through Daugman's filter), normalization (for creating rectangular block of fixed size through rubber sheet model), image enhancement (to convert low contrast image to high contrast image and minimize non-uniform illumination by applying Gabor filter) and image matching (to perform template matching region and different iris images are represented as with the help of Hamming distance). The main advantage of the proposed technique is that promise accuracy and performance can be achieved even if images are taken from a distance.

\section{CONCLUSIONS}

Iris recognition is one of most effective method for identification of human being. This paper provides review on various existing methods proposed by different researchers from time to time for iris recognition. There are three main stages in iris recognition system: image preprocessing, feature extraction and template matching. A literature review of the most prominent algorithms implemented in each stage is presented.

For the purpose of research and development of Iris recognition technology there are few public and freely available databases to have sample images. These iris databases contributes rich amount of iris images which were taken in different environments. In this paper we have discussed about the publicly available iris image datasets.

\section{ACKNOWLEDGMENT}

The authors would like to welcome the reviewers for their critical and constructive comments and suggestions. The authors would like to thank the Authorities of National Institute of Electronics and InformatioTechnology, Dr. Babasaheb Ambedkar Marathwada University, Aurangabad for providing the infrastructure to carry out the research.

\section{REFERENCES}

[1] S. Prabhakar, S. Pankanti, and A. K. Jain, "Biometric recognition: Security and privacy concerns", IEEE Security and Privacy, vol. 1, No. 2, pp. 33-42, 2003.

[2] Ravi Das, "Retinal recognition Biometric technology in practice", Keesing Journal of Documents \& Identity, vol. 22, pp. 11-14, 2007.

[3] Charles Boncelet (2005)."Image Noise Models". In Alan C. Bovik. Handbook of Image and Video Processing.

[4] Priyanka Shrivastava, Uday Pratap Singh, "Noise Removal using First Order Neighborhood Mean Filter", 978-1-4799-3064-7/14/\$31. 00, 2014 IEEE.

[5] Fu Bin, Xiong XinyanI, Sun Guozhen, "An efficient Mean Filter Algorithm”,, Proceedings of the 2011 IEEEIICME International Conference on Complex Medical Engineering May 22 - 25, Harbin, China.

[6] Yi Hong, Sam Kwong, Hanli Wang, "Decision-Based Median Filter Using K-Nearest Noise-Free Pixels", ICASSP 2009, 978-1-42442354-5/09/\$25.00,2009 IEEE.

[7] B. Sravani, M. V. N ageswara Rao, "Removing of high density salt and pepper noise using Fuzzy Median Filter", 978-1-47995958-7114/\$31.00, 2014 IEEE.

[8] Jacob Benesty, Jingdong Chen, and Yiteng (Arden) Huang, "Study of the Widely Linear Wiener Filter for Noise Reduction", ICASSP 2010, 978-1-4244-4296-6/10/\$25.00, 2010 IEEE.

[9] SmitTrambadia, Paresh Dholakia, "Design and Analysis of an Image Restoration Using Wiener Filter with a Quality Based Hybrid Algorithms", IEEE Sponsored 2nd International Conference on Electronics and Communication System (ICECS 2015), 978-14788-7225-8/15/\$31.00,2015 IEEE.

[10] Peng Xuange,Xiao Ying, Zhu Bing, Liu Yong, "River Sediment image denoising algorithm based on multidirectional 1D wavelet Transform”, 2009 International Conference on Information Management, Innovation Management and Industrial Engineering, 9780-7695-3876-1/09 \$25.00, 2009 IEEE DOI 10.1109/ICIII.2009.215

[11] Hong Yang,Yiding Wang, "An Improved Method of Wavelets Basis Image Denoising Using Besov Norm Regularization”, Fourth International Conference on Image and Graphics, 0-7695-2929-1/07 \$25.00, 2007 IEEE DOI 10.1109/ICIG.2007.143 
[12] Awanish Kumar Shukla,R. L. Verma, Mohd. Sanawer Alam, Vikrant Bhateja, "Directional Order Statistics Filtering for Suppression of Salt and Pepper Noise", 2014 International Conference on Signal Processing and Integrated Networks (SPIN). 978-1-4799-28668/14/\$31.00, 2014 IEEE.

[13] Bram Cornelis, Marc Moonen, and Jan Wouters, "Performance Analysis of Multichannel Wiener Filter-Based Noise Reduction in Hearing Aids Under Second Order Statistics Estimation Errors", IEEE Transactions On Audio, Speech, And Language Processing, VOL. 19, NO. 5, JULY 2011

[14] 14.Fraz,P.Remagnino,A.Hoppe,B.Uyyanonvara,A.Rudnicka,C.Owen,Barman, "Blood vessel segmentation methodologies in retinal images - a survey", Computer Methods and Programs in Biomedicine.

[15] Liu, Y.Sun, "Recursive tracking of vascular networks in angiograms based on the detection-deletion scheme", IEEE Transactions on Medical Imaging 12 (2) (1993) 334-341.

[16] L.Zhou, M.S.Rzeszotarski, L.J.Singerman, J.M.Chokreff, "The detection and quantification of retinopathy using digital angiograms", IEEE Transactions on Medical Imaging 13(4)(1994)619-626.

[17] O.Chutatape,L.Zheng,S.Krishnan, "Retinal blood vessel detection and tracking by matched Gaussian and kalman filters", Engineering in Medicine and Biology Society, 1998., Proceedings of the $20^{\text {th }}$ Annual International Conference of the IEEE,vol.6, IEEE,1998,pp.3144-3149.

[18] A.Tolias, S.M.Panas, "A fuzzy vessel tracking algorithm for retinal images based on fuzzy clustering", IEEE Transactions on MedicalImaging17 (2) (1998) 263-273.

[19] A.Can, H.Shen, J.N.Turner, H.L.Tanenbaum, B.Roysam, "Rapid automated tracing and feature extraction from retinal fundus images using direct exploratory algorithms", IEEE Transactions on Information Technology in Biomedicine 3(2) (1999)125-138.

[20] Y. Yin, M. Adel, S. Bourennane, "Retinal vessel segmentation using a probabilistic tracking method", Pattern Recognition 45 (2012) $1235-1244$.

[21] S. Chaudhuri, S. Chatterjee, N. Katz, M. Nelson, M. Goldbaum," Detection of blood vessels in retinal images using two-dimensional matched filters", IEEE Transactions on Medical Imaging 8 (3) (1989) 263-269.

[22] A. Hoover, V. Kouznetsova, M. Goldbaum," Locating blood vessels in retinal images by piecewise threshold probing of a matched filter response", IEEE Transactions on Medical Imaging 19 (3) (2000) 203-210.

[23] M. Al-Rawi, M. Qutaishat, M. Arrar," An improved matched filter for blood vessel detection of digital retinal images", Computers in Biology and Medicine 37 (2) (2007) 262-267.

[24] L. Zhang, Q. Li, J. You, D. Zhang," A modified matched filter with double-sided thresholding for screening proliferative diabetic retinopathy", IEEE Transac- tions on Information Technology in Biomedicine 13 (4) (2009) 528-534.

[25] B. Zhang, L. Zhang, F. Karray, "Retinal vessel extraction by matched filter with first-order derivative of gaussian", Computers in Biology and Medicine 40 (4) (2010) 438-445.

[26] C. Sinthanayothin, J.F. Boyce, H.L. Cook, T.H. Williamson, "Automated localisation of the optic disc, fovea, and retinal blood vessels from digital colour fundus images", British Journal of Ophthalmology 83 (8) (1999) 902.

[27] 27.Niemeijer,J.Staal,B.vanGinneken,M.Loog,M.D.Abramoff,"Comparative study of retinal vessel segmentation methods on a new publicly available database", in: Proceedings of SPIE,vol.5370,2004,pp.648-656.

[28] 28. M.Sofka,C.V.Stewart, "Retinal vessel center line extraction using multiscale matched filters, confidence and edge measures" ,IEEE Transactions on Medical Imaging 25(12)(2006)1531-1546.

[29] 29.C.A.Lupascu, D.Tegolo,E.Trucco, "Fabc:retinal vessel segmentation using adaboost", IEEE Transactions on Information Technology in Biomedicine 14(5) (2010) 1267-1274.

[30] 30.D.Marin,A.Aquino,M.E.Arias, J.M.Bravo,"A new supervised method for blood vessel segmentation in retinal images by using gray-level and moment invariants-based features", IEEE Transactions on Medical Imaging 30(1)(2011)146-158.

[31] 31. X.You,Q.Peng,Y.Yuan,Y.Cheung,J.Lei, "Segmentation of retinal blood vessels using the radial projection and semi-supervised approach", Pattern Recognition 44(2011)2314-2324.

[32] 32. Li Guodong, Xiang Dehui, Yang Fei, Wan Xiaonan, Yang Xin, Tian Jie, "Automatic detection of retinal vascular bifurcations and crossovers based on isotropy and anisotropy ", Proc. SPIE 8669, Medical Imaging 2013: Image Processing, 86691T (March 13, 2013); Doi:10.1117/12.2006710;

[33] 33.Azzopardi George, and Petkov Nicolai, "Detection of Retinal Vascular Bifurcations by Trainable V4-Like Filters", A. Berciano et al. (Eds.): CAIP 2011, LNCS 6854, pp. 451-459, 2011, Springer-Verlag Berlin Heidelberg 2011.

[34] 34. Bhuiyan A., Nath B., Chua J., Ramamohanarao K., "Automatic Detection of Vascular Bifurcations and Crossovers from Color Retinal Fundus Images" SignalImage Technologies and Internet-Based System, 2007. SITIS '07. Third International IEEE Conference on Signal-Image Technologies and Internet-Based System, pp: 711 - 718, 2007.

[35] 35. Ardizzone Edoardo, Pirrone Roberto, Gambino Orazio, and Scaturro Francesco, " Automatic Extraction of Blood Vessels, Bifurcations and End Points in the Retinal Vascular Tree", 13th International Conference on Biomedical Engineering, IFMBE Proceedings Volume 23, pp 22-26, 2009.

[36] Rishabh Parashar, Sandeep Joshi, "Comparative Study of Iris Databases and UBIRIS Database for Iris Recognition Methods for NonCooperative Environment”, International Journal of Engineering Research \& Technology (IJERT), Vol. 1, Issue 5, July - 2012.

[37] Casia Iris Database: http://www.idealtest.org/findDownloadDbByMode.do?mode=Iris

[38] Multimedia Iris Database: http://wwwl.mmu.edu.my/ ccteo/

[39] Upol Iris Database: http://phoenix.inf.upol.cz/iris/

[40] Ubiris Iris Database: http://iris.di.ubi.pt/

[41] IIT Delhi Database: http://www4.comp.polyu.edu.hk/ csajaykr/IITD/Database_Iris.htm

[42] C.H. Daouk et al., "IRIS RECOGNITION", Proceeding of the IEEE ISSPIT, Marrakesh, pp. 558-562, 2002.

[43] S.I. Noh et al., "A Novel Method to Extract Features for Iris Recognition System", Audio- and Video-Based Biometric Person Authentication, Springer, pp. 862-868, 2003.

[44] G. Xu, Z. Zhang and Y. Ma, "Improving The Performance Of Iris Recognition System Using Eyelids And Eyelashes Detection and Iris Image Enhancement", Proceeding of the IEEE International Conference on Cognitive Informatics, pp. 871-876, 2006.

[45] J. Daugman, "New Methods in Iris Recognition", IEEE Transactions on Systems, Man and Cybernetics, Vol. 37, No. 5, pp. $1167-$ 1175, 2007.

[46] A. Azizi and H.R. Pourreza, "Efficient IRIS RecognitionThrough Improvement of Feature Extraction and subset Selection", International Journal of Computer Science and Information Security, Vol. 2, No. 1, June 2009.

[47] S.V. Sheela and P.A. Vijaya, "Iris Recognition Methods -Survey", International Journal of Computer Applications, Vol. 3, No. 5, pp. 19-25, June 2010.

[48] Gupta, R. and H. Saini, 2011. Generation of Iris Template for recognition of Iris in efficient Manner, International Journal of Computer Science and Technologies (IJCSIT), 2(4). 